\title{
Integrating Interference Frequency Components Elicited by Monitor Refresh Rate to Enhance Frequency Detection of SSVEPs
}

\author{
Masaki Nakanishi*, Student Member, IEEE, Yijun Wang, Member, IEEE, Yu-Te Wang, Student Member, IEEE, \\ Yasue Mitsukura, Member, IEEE, and Tzyy-Ping Jung, Senior Member, IEEE
}

\begin{abstract}
Steady-state visual evoked potential (SSVEP)based brain-computer interfaces (BCIs) have potential to realize a direct communication between the human brain and the outside environment in real-life situations. Recently, we proposed a stimulation approximation approach to increase the number of visual stimuli that can be realized on a computer monitor. In addition to the fundamental and harmonic frequencies, the refresh rate-based stimulation approach also elicits SSVEPs at other frequencies that are termed interference frequencies, which are derived from the interaction between the stimulation frequency and the refresh rate. This study aims to investigate properties of the interference frequency components, and propose to integrate the interference frequency components to enhance frequency detection of SSVEPs. The results suggest that the proposed approach could substantially improve the frequency detection accuracy of SSVEPs.
\end{abstract}

\section{INTRODUCTION}

Steady-state visual evoked potential (SSVEP) is the electrical response of brain elicited by repetitive visual stimulation. It has been widely used in electroencephalogram (EEG)-based brain-computer interfaces (BCIs) due to its advantages of little user training, ease of use, and high information transfer rate (ITR) [1]-[5]. In an SSVEP-based $\mathrm{BCI}$, users are asked to fixate on one of multiple visual stimuli flickering at different frequencies, and the target visual stimulus, which a user is gazing at, can be recognized as the command of an interface through analyzing the SSVEPs. In this way, an SSVEP-based BCI can directly translate intentional brain activities to the commands tagged by stimulus frequencies to control an output device.

Visual stimulus design plays an important role in an SSVEP-based BCI [3]. Visual stimuli can be presented using flashing light-emitting diodes (LEDs) or flickers on a computer screen such as cathode ray tube (CRT) monitor or liquid crystal display (LCD) monitor [6]. Compared with the LED based stimulator, the stimulation parameters such as the amount, color, pattern, size, and position of visual stimuli can be configured more flexibly when using a computer monitor. However, the number of frequencies that can be

*Research is supported in part by a gift fund from the Swartz Foundation, US Army Research Laboratory, Army Research Office, DARPA and Office of Naval Research. M. Nakanishi is also supported by Japan Society for the Promotion of Science.

*M. Nakanishi and Y. Mitsukura are with Graduate School of Science and Technology, Keio University, 3-14-1, Hiyoshi, Kohoku, Yokohama, Kanagawa, 223-8522 Japan. (e-mail: ñakanishi@mitsu.sd.keio.ac.jp, mitsukura@sd.keio.ac.jp)

Y. Wang, Y. -T. Wang and T. -P. Jung are with Swartz Center for Computational Neuroscience, Institute for Neural Computation, University of California, San Diego, La Jolla, CA92093 USA. (e-mail: yijun@ sccn.ucsd.edu, ŷtwang@ucsd.edu, jung@sccn.ucsd.edu) presented is always limited by the refresh rate because the number of frames in a stimulus period has to be a constant in the conventional frame based stimulus design. In this case, some applications, which require a large number of commands, cannot be implemented. Generally, the increase of the number of commands can lead to an increase of the ITR [3]. Therefore, it is of great importance to find a solution to render flickering stimuli with a high frequency resolution on a computer monitor.

Recently, Wang et al. proposed a method to realize visual stimulus presentation on a computer monitor for eliciting SSVEPs with a high frequency resolution by approximating a flickering rate [7]. They obtained an averaged ITR of $75.4 \mathrm{bits} / \mathrm{min}$ in an online SSVEP-based BCI test. The efficacy of the approach was also proved by other online BCI experiments [8]. In addition, a recent study performed a quantitative comparison between the SSVEPs elicited by the approximation approach and the constant-period approach [9]. In general, the SSVEPs have the same frequency components as the fundamental and harmonics of the stimulating frequency. However, when using the approximation approach, the SSVEPs also have components at additional frequencies that are termed interference frequencies (i.e., indicating the interference between the stimulating frequency and the refresh rate in this study). For example, a $10 \mathrm{~Hz}$ stimulus signal under a $75 \mathrm{~Hz}$ refresh rate includes not only the fundamental component at $10 \mathrm{~Hz}$, the second and third harmonics at $20 \mathrm{~Hz}$ and $30 \mathrm{~Hz}$, but also components at additional frequencies such as $5 \mathrm{~Hz}, 15 \mathrm{~Hz}$, and $25 \mathrm{~Hz}$ (See Fig. 1). Therefore, the interference frequencies could provide additional information for frequency detection of the SSVEPs. To our knowledge, currently, no study has investigated the characteristics of the SSVEPs at the interference frequencies, and they were totally ignored in previous BCI studies [7]-[9]. This study aims to analyze the amplitude spectrum and scalp distribution of SSVEPs at the interference frequencies, and develop a new strategy to integrate these frequency components to enhance frequency detection of SSVEPs elicited by monitor refresh rate.

\section{Methods}

\section{A. Visual Stimuli}

In the conventional constant-period approach, the number of frames in a period is a constant. For instance, a $10 \mathrm{~Hz}$ visual stimulus under a $60 \mathrm{~Hz}$ refresh rate can be realized by the reversing stimulus pattern between black and white every three frames. However, a flickering frequency by which the 
Flickering signal at $75 \mathrm{~Hz}$ refresh rate

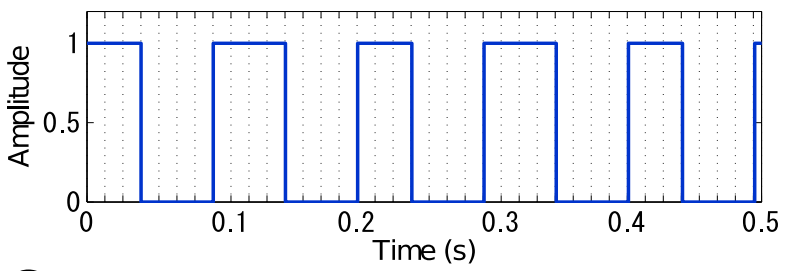

C

Flickering signal at $120 \mathrm{~Hz}$ refresh rate

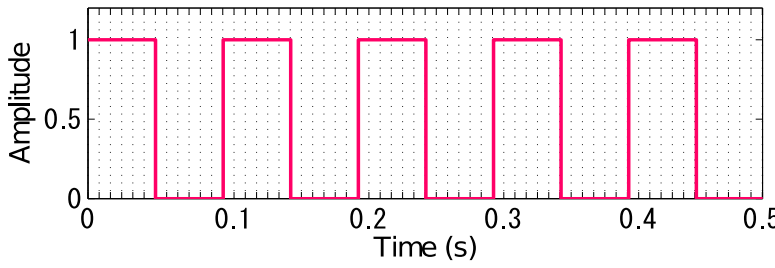

B

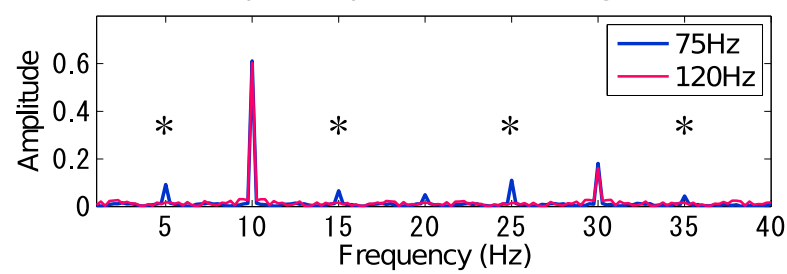

$\mathrm{D}$

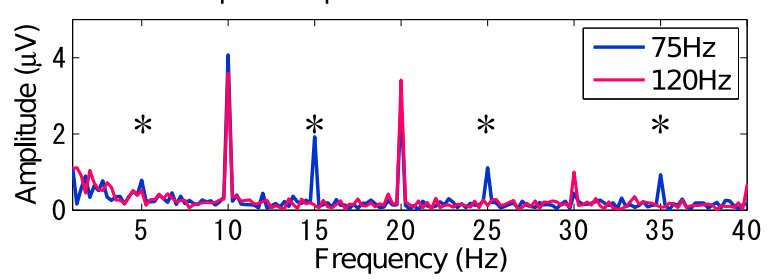

Fig. 1. Time series flickering signals at $10 \mathrm{~Hz}$ presented on a computer screen with (A) $75 \mathrm{~Hz}$ and (C) $120 \mathrm{~Hz}$ refresh rate, (B) the amplitude spectra of flickering signals, and (D) the amplitude spectra of elicited SSVEPs for a sample subject. The asterisks indicate the interference frequencies.

refresh rate is not dividable (i.e., $11 \mathrm{~Hz}$ ) cannot be realized in this way. The approximation approach proposed in [7] can realize such flickering frequency by using a varying number of frames in a period. For example, $11 \mathrm{~Hz}$ can be realized by mixing two types of periods with five or six frames in a period as '1110001110011100011000111...', where '1' and ' 0 ' represents a 'black' and 'white' frame respectively. Based on this approach, the stimulus sequence $c(f, i)$ corresponding to frequency $f$ can be generated by the following equation:

$$
c(f, i)=\text { square }\left[2 \pi f \frac{i}{\text { RefreshRate }}\right]
$$

where $i$ indicates the frame index. In this way, a stimulus sequence at any frequency up to half of the refresh rate can be generated. Fig. 1(B) illustrates the amplitude spectra of the stimulus signals at $10 \mathrm{~Hz}$ generated by the approximation approach and the constant-period approach under $75 \mathrm{~Hz}$ and $120 \mathrm{~Hz}$ refresh rates, respectively.

\section{B. EEG Aquisition}

The visual stimulus was a $5 \times 5 \mathrm{~cm}$ square rendered at the center of a 21 -inch CRT monitor with $75 \mathrm{~Hz}$ and $120 \mathrm{~Hz}$ refresh rates. The stimulus frequencies ranged from $9 \mathrm{~Hz}$ to $13 \mathrm{~Hz}$ with an interval of $1 \mathrm{~Hz}$. Here, all the visual stimuli were generated by (1). The stimulus program was developed in Microsoft Visual C++ using the Microsoft DirectX 9.0 framework and rendered on Windows XP platform.

Ten healthy subjects with normal or corrected-to-normal vision participated in this experiment. Each subject was seated in a comfortable chair in front of the monitor and asked to fixate on the visual stimulus presented at the center of the monitor for 30 seconds. The experiment consisted of four sessions, each including ten 30s-long trials for the five different stimulus frequencies. To avoid visual fatigue, there was a several-second break between two consecutive trials and a several-minute break between two sessions. The order of the frequencies was randomized. EEG data were recorded using $\mathrm{Ag} / \mathrm{AgCl}$ electrodes from 256 locations distributed over the entire head using a BioSemi ActiveTwo EEG system (Biosemi, Inc.). Electrode locations were measured with a 3-D digitizer system (Polhemus, Inc.). All signals were amplified and digitized at a sampling rate of $2048 \mathrm{~Hz}$. All electrodes were referred to the CMS electrode close to $\mathrm{Cz}$.

\section{EEG Processing}

The 256-channel EEG data were first down-sampled to $256 \mathrm{~Hz}$. For each 30s-long trial, six 4s-long EEG epochs were extracted according to event triggers generated by the stimulus program. For each stimulus frequency, the epochs from all sessions were put together to form a dataset comprising 24 epochs.

This study first investigated the amplitude of SSVEPs calculated by fast Fourier transform (FFT) and its scalp distribution. The scalp topography maps based on multichannel SSVEP amplitudes are illustrated using the TOPOPLOT function in EEGLAB toolbox [10]. This study also performed offline classification of SSVEPs at all five frequencies using a canonical correlation analysis (CCA)-based method, which has been widely used in SSVEP-based BCIs [11], [12]. CCA is a statistical way to measure the linear relationship between two multidimensional variables, which may have some underlying correlation. CCA has been widely used in an SSVEP-based BCI. Considering two multidimensional variable $X, Y$ and their linear combinations $x=X^{T} W_{x}$ and $y=Y^{T} W_{y}$, CCA finds the weight vectors, $W_{x}$ and $W_{y}$, which maximize the correlation between $x$ and $y$ by solving the following problem:

$$
\max _{W_{x}, W_{y}} \rho(x, y)=\frac{E\left[W_{x}^{T} X Y^{T} X_{y}\right]}{\sqrt{E\left[W_{x}^{T} X X^{T} W_{x}\right] E\left[W_{y}^{T} Y Y^{T} W_{y}\right]}} .
$$

The maximum of $\rho$ with respect to $W_{x}$ and $W_{y}$ is the maximum canonical correlation. Projections onto $W_{x}$ and $W_{y}$ are called canonical variants. Here, $X$ refers to the set 
TABLE I

INTERFERENCE FREQUENCIES FOR EACH STIMULUS

\begin{tabular}{lllll}
\hline \multirow{2}{*}{$\begin{array}{l}\text { Stimulus } \\
\text { frequency }(\mathrm{Hz})\end{array}$} & \multicolumn{4}{l}{ Interference } \\
\cline { 2 - 5 } & 1st & 2nd & 3rd & 4th \\
\hline 9 & 6 & 12 & 24 & 30 \\
10 & 5 & 15 & 25 & 35 \\
11 & 7 & 15 & 30 & 36 \\
12 & 9 & 15 & 33 & 39 \\
13 & 10 & 16 & 36 & 42 \\
\hline
\end{tabular}

of 4s-long multi-channel EEG signals and $Y$ refers to the set of reference signals that have the same length as $X$. To avoid overfitting, sixteen electrodes over the occipital region were selected for CCA. The reference signals $Y_{f}$ were set as

$$
Y_{f}=\left[\begin{array}{c}
\sin (2 \pi f t) \\
\cos (2 \pi f t) \\
\vdots \\
\sin \left(2 \pi N_{h} f t\right) \\
\cos \left(2 \pi N_{h} f t\right)
\end{array}\right]
$$

where $f$ is the target frequency, $N_{h}$ is the number of harmonics, and $N$ is the number of sampling points. To recognize the frequency of the SSVEPs, CCA calculates the canonical correlation between the multi-channel EEG signals and the reference signals at each stimulus frequency. The frequency of the reference signals with the maximal correlation was selected as the frequency of the SSVEPs.

In this study, the interference frequency components were included in the reference signals to improve the frequency identification accuracy:

$$
Y_{f}=\left[\begin{array}{c}
\sin (2 \pi f t) \\
\cos (2 \pi f t) \\
\vdots \\
\sin \left(2 \pi N_{h} f t\right) \\
\cos \left(2 \pi N_{h} f t\right) \\
\sin \left(2 \pi f_{i_{1}} t\right) \\
\cos \left(2 \pi f_{i_{1}} t\right) \\
\vdots \\
\sin \left(2 \pi f_{i_{M}} t\right) \\
\cos \left(2 \pi f_{i_{M}} t\right)
\end{array}\right]
$$

where, $f_{i_{m}}, m=1,2, \ldots, M$ is the $m$-th interference frequency. The interference frequencies are defined as the frequencies with high amplitude except for the stimulus frequency and its harmonics in the stimulus signal. Table I lists the interference frequencies for each stimulus frequency (9 to $13 \mathrm{~Hz}$ ).

\section{RESULTS}

Fig.1(B) shows that the stimulus signal at $10 \mathrm{~Hz}$ under the $75 \mathrm{~Hz}$ refresh rate has frequency components at interference frequencies with much higher amplitude than that under the $120 \mathrm{~Hz}$ refresh rate. Under the $75 \mathrm{~Hz}$ refresh rate, the SSVEPs includes not only the fundamental and second harmonics at $10 \mathrm{hz}$ and $20 \mathrm{~Hz}$, but also components at $5 \mathrm{~Hz}, 15 \mathrm{~Hz}$,
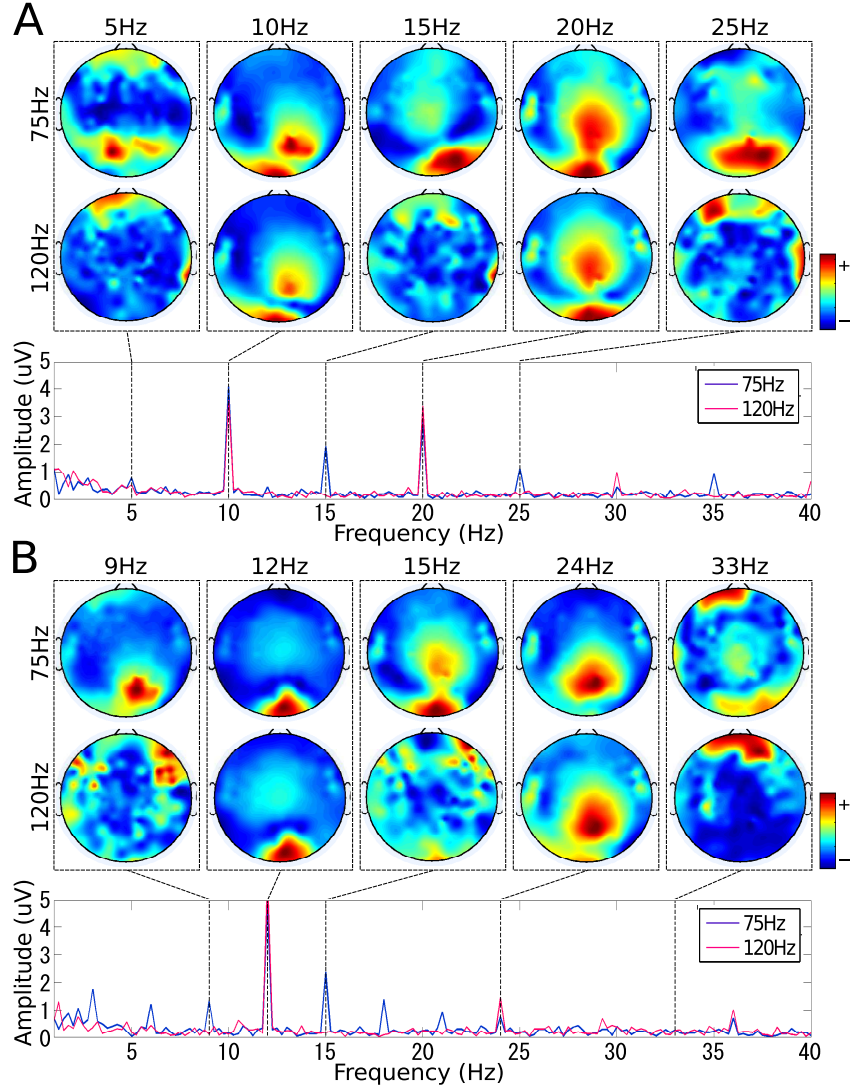

Fig. 2. The amplitude spectrum and scalp topographies of SSVEPs at (A) $10 \mathrm{~Hz}$ and (B) $12 \mathrm{~Hz}$ under $75 \mathrm{~Hz}$ and $120 \mathrm{~Hz}$ refresh rates for subject 2 .

25Hz,and 35Hz (see Fig.2(D)). Fig.2 compares the amplitude spectra of elicited SSVEPs at the $\mathrm{Oz}$ electrode and scalp topographies of EEG signals at the stimulus frequency $(10 \mathrm{~Hz}$ and $12 \mathrm{~Hz}$ ), second harmonics, and interference frequencies between the two stimulation conditions. For the SSVEPs elicited by the $10 \mathrm{~Hz}$ stimulation, $10 \mathrm{~Hz}$ and $20 \mathrm{~Hz}$ indicate the fundamental and second harmonics of the stimulating frequency respectively, and $5 \mathrm{~Hz}, 15 \mathrm{~Hz}$ and $25 \mathrm{~Hz}$ indicate the interference frequencies. For the SSVEPs elicited by the $12 \mathrm{~Hz}$ stimulus, $12 \mathrm{~Hz}$ and $24 \mathrm{~Hz}$ indicate the fundamental and second harmonics, and $9 \mathrm{~Hz}, 15 \mathrm{~Hz}$ and $33 \mathrm{~Hz}$ indicate the interference frequencies. The scalp topographies show that the electrodes at the occipital area have the highest amplitude at all harmonic and interference frequencies under the $75 \mathrm{~Hz}$ refresh rate. However, the SSVEPs only show strong activities at the fundamental and second harmonics under the $120 \mathrm{~Hz}$ refresh rate.

Table II lists the offline classification accuracy for five stimulus frequencies (under the $75 \mathrm{~Hz}$ refresh rate) using the conventional CCA-based method and the proposed method. In this study, three methods including the CCA-based method with the fundamental harmonic (Conventional 1), the CCAbased method with the fundamental and second harmonics (Conventional 2), and the proposed method (Proposed) were used to estimate the classification accuracy. Here, both $N_{h}$ and $M$ were set to 2 in the proposed method, and the 
TABLE II

TARGET IDENTIFICATION ACCURACY (\%)

\begin{tabular}{llll}
\hline Subject & Conventional 1 & Conventional 2 & Proposed \\
\hline s1 & 78.40 & 81.60 & 84.00 \\
s2 & 82.68 & 83.46 & 88.18 \\
s3 & 100.00 & 100.00 & 100.00 \\
s4 & 66.67 & 70.54 & 82.17 \\
s5 & 96.80 & 97.60 & 97.60 \\
s6 & 99.19 & 99.19 & 99.19 \\
s7 & 100.00 & 100.00 & 100.00 \\
s8 & 89.15 & 89.92 & 89.92 \\
s9 & 84.80 & 88.80 & 93.60 \\
s10 & 94.35 & 94.35 & 93.55 \\
Mean \pm STD & $89.20 \pm 11.07$ & $90.55 \pm 9.73$ & $92.82 \pm 6.57$ \\
\hline
\end{tabular}

interference frequencies were selected so as to maximize the classification accuracy. The averaged accuracy of Conventional 1 and Conventional 2 was $89.20 \%$ and $90.55 \%$, respectively. The accuracy of the proposed method was $92.82 \%$, which was significantly higher than the conventional methods (Conventional 1 vs. Proposed: $p=0.03$, Conventional 2 vs. Proposed: $p=0.048)$. There was significant difference between the two conventional methods $(p=0.02)$.

Fig.3 shows the classification accuracy obtained using the conventional and proposed methods with different data length $T$ (from 1 to 4 seconds). For all conditions, the proposed method obtained the highest classification accuracy. There was a significant difference between the accuracies of the Proposed and Conventional 1 methods $(T=1: p=0.003$, $T=2: p=0.004, T=3: p=0.02$, and $T=4: p=0.03$ ), and the accuracies of the Proposed and Conventional 2 methods $(T=1$ : $p=0.01, T=2: p=0.01, T=3: p=0.03$, and $T=4: p=0.048$ ). These results indicate that, by considering the interference frequencies in the frequency detection, the proposed method can significantly improve the frequency detection accuracy.

\section{DISCUSSIONS AND CONCLUSIONS}

The efficacy of the stimulus presentation based on the approximation approach has been reported in previous studies [7]-[9]. However, the interference frequency components in SSVEPs have not been investigated and were ignored in

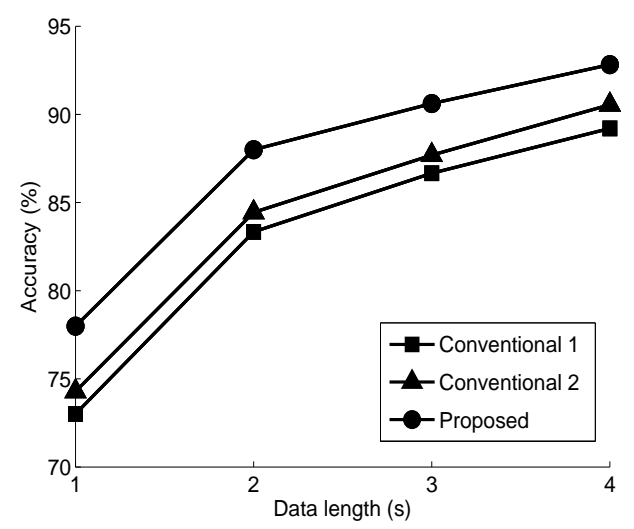

Fig. 3. The amplitude spectrum and scalp topographies of SSVEP at $10 \mathrm{~Hz}$ for subject 4 . these studies. This study first pointed out that the interference frequencies in SSVEPs should be considered in stimulus design and data analysis. This study showed distinct spectral and spatial characteristic of the SSVEP components at the interference frequencies elicited by the visual stimuli generated by the approximation approach. This study also classified the SSVEPs at frequencies from $9 \mathrm{~Hz}$ to $13 \mathrm{~Hz}$ with an interval of $1 \mathrm{~Hz}$, and compared the classification performance among the conventional and proposed CCA-based methods. By considering the interference frequency components, the CCAbased classification method can substantially improve the accuracy of frequency detection. Further investigations are required to explore the relationship between the amplitudes of the stimulus signal and the SSVEPs at the interference frequencies.

The approximation approach for generating SSVEP stimulus is essential to implement a practical BCI system that requires a large number of user selections such as an 8target cursor system, a 12-target phone dialing system, and a 30-target spelling system. Our future work will focus on the implementation of a multi-command, real-time, and portable BCI system using the proposed method that considers the interference frequency components in SSVEPs.

\section{REFERENCES}

[1] J. R. Wolpaw, N. Birbaumer, D. J. McFarland, G. Pfurtscheller, and T. M. Vaughan, "Brain-computer interfaces for communication and control," Clinical Neurophysiology, vol. 113, pp. 767-791, 2002.

[2] M. Cheng, X. Gao, S. Gao, and D. Xu, "Design and Implementation of a Brain-Computer Interface With High Transfer Rates," IEEE Transactions on Biomedical Engineering, vol. 49, pp. 1181-1186, 2002.

[3] Y. Wang, X. Gao, B. Hong, C. Jia, and S. Gao, "Brain-computer interfaces based on visual evoked potentials: feasibility of practical system design," IEEE Engineering in Medicine and Biology Magazine, vol. 27, no. 5, pp. 64-71, 2008.

[4] P.-L. Lee, C.-H. Wu, J.-C. Hsieh, and Y.-T. Wu, "Visual evoked potential actuated brain computer interface: a brain-actuated cursor system," Electronics Letters, vol. 41, pp. 832-834, 2005.

[5] A. Materka and N. Byezuk, "Alternate half-field stimulation technique for SSVEP-based brain-computer interfaces," Electronics Letters, vol. 42, pp. 321-322, 2006.

[6] Z. Wu, Y. Lai, Y. Xia, D. Wu, and D. Yao, "Stimulator selection in SSVEP-based BCI," Medical Engineering and Physics, vol. 30, pp. 1079-1088, 2008.

[7] Y. Wang, Y.-T. Wang, and T.-P. Jung, "Visual stimulus design for high-rate SSVEP," Electronics Letters, vol. 46, no. 15, pp. 1057-1058, 2010.

[8] Y.-T. Wang, Y. Wang, and T.-P. Jung, "A Cell-phone-based Brain Computer Interface for Communication in Daily Life," Journal of Neural Enginneering, vol. 8, p. 025018, 2011.

[9] M. Nakanishi, Y. Wang, Y.-T. Wang, Y. Mitsukura, and T.-P. Jung, "An approximation approach for rendering visual flickers in SSVEPbased BCI using monitor refresh rate," in 35th Annual International Conference of the IEEE Engineering in Medicine and Biology Society, July 2013.

[10] A. Delorme and S. Makeig, "EEGLAB: an open source toolbox for analysis of single-trial EEG dynamics including independent component analysis," Journal of Neuroscience Methods, vol. 134, no. 1, pp. 9-21, 2004

[11] Z. Lin, C. Zhang, W. Wu, and X. Gao, "Frequency recognition based on canonical correlation analysis for SSVEP-based BCIs," IEEE Transaction on Biomedical Engineering, vol. 54, no. 6, pp. 1172-1176, 2007.

[12] G. Bin, X. Gao, Z. Yan, B. Hong, and S. Gao, "An online multi-channel SSVEP-based brain-computer interface using a canonical correlation analysis method," Journal of Neural Engineering, vol. 6, p. 046002, 2009. 